\title{
Manganese ferrite-based nanoparticles induce ex vivo, but not in vivo, cardiovascular effects
}

\author{
This article was published in the following Dove Press journal: \\ International Journal of Nanomedicine \\ 8 July 2014 \\ Number of times this article has been viewed
}

\section{Allancer DC Nunes \\ Laylla S Ramalho \\ Álvaro PS Souza' \\ Elizabeth P Mendes ${ }^{1,3}$ \\ Diego B Colugnati' \\ Nícholas Zufelato² \\ Marcelo H Sousa ${ }^{4}$ \\ Andris F Bakuzis ${ }^{2}$ \\ Carlos H Castrol,3}

'Department of Physiological Sciences, ${ }^{2}$ Physics Institute, Federal University of Goiás, Goiânia, Brazil; ${ }^{3}$ National Institute of Science and Technology in Nanobiopharmaceutics, Belo Horizonte, Brazil; ${ }^{4}$ Faculty of Ceilândia, University of Brasília, Brasília-DF, Brazil
Correspondence: Carlos Henrique

Castro

Departamento de Ciências Fisiológicas, ICB II, Universidade Federal de Goiás,

Campus II, 7400 I-970, Goiânia, GO, Brazil

Tel +55 $62352 I \quad 1775$

Fax +55 623521 I204

Email castro@ufg.br
Abstract: Magnetic nanoparticles (MNPs) have been used for various biomedical applications. Importantly, manganese ferrite-based nanoparticles have useful magnetic resonance imaging characteristics and potential for hyperthermia treatment, but their effects in the cardiovascular system are poorly reported. Thus, the objectives of this study were to determine the cardiovascular effects of three different types of manganese ferrite-based magnetic nanoparticles: citrate-coated (CiMNPs); tripolyphosphate-coated (PhMNPs); and bare magnetic nanoparticles (BaMNPs). The samples were characterized by vibrating sample magnetometer, X-ray diffraction, dynamic light scattering, and transmission electron microscopy. The direct effects of the MNPs on cardiac contractility were evaluated in isolated perfused rat hearts. The CiMNPs, but not PhMNPs and BaMNPs, induced a transient decrease in the left ventricular end-systolic pressure. The PhMNPs and BaMNPs, but not CiMNPs, induced an increase in left ventricular end-diastolic pressure, which resulted in a decrease in a left ventricular end developed pressure. Indeed, PhMNPs and BaMNPs also caused a decrease in the maximal rate of left ventricular pressure rise $(+\mathrm{dP} / \mathrm{dt})$ and maximal rate of left ventricular pressure decline $(-\mathrm{dP} / \mathrm{dt})$. The three MNPs studied induced an increase in the perfusion pressure of isolated hearts. BaMNPs, but not PhMNPs or CiMNPs, induced a slight vasorelaxant effect in the isolated aortic rings. None of the MNPs were able to change heart rate or arterial blood pressure in conscious rats. In summary, although the MNPs were able to induce effects ex vivo, no significant changes were observed in vivo. Thus, given the proper dosages, these MNPs should be considered for possible therapeutic applications.

Keywords: cardiac function, isolated heart, magnetic fluids, magnetic nanoparticles, nanomedicine

\section{Introduction}

There has been an exponential increase in the use of nanotechnology for medical applications over the past few decades. Nanoparticles are defined as having a nominal diameter smaller than $100 \mathrm{~nm}$ and are recognized as an important class of newly engineered materials with a wide range of biomedical applications. Nanoparticles have recently been used for the diagnosis and treatment of diseases, including cardiovascular diseases. ${ }^{1-3}$

Magnetic fluids, generally composed of stable dispersions of magnetic nanoparticles (MNPs) in a liquid carrier, ${ }^{4}$ represent very good candidates for these purposes. This is a result of their application in the diagnosis of diseases as contrast agents ${ }^{5}$ for cancer treatment through magnetic hyperthermia, ${ }^{6}$ biological detection, ${ }^{7}$ stem cell labeling, ${ }^{8}$ and drug delivery. ${ }^{9,10}$

A wide variety of MNPs have been studied, with differing size and type of coating material, including dextran, citrate, phosphate, albumin, and silicones. These nanoparticles can be encapsulated into different compartments, such as liposomes, polymeric nanospheres, nanocapsules, and dendrimers, among others. ${ }^{1-14}$ When adequately 
coated, nanocarriers can increase the time of circulation (polyethylene glycol) or improve active targeting (peptides and antibodies) of nanoparticles. ${ }^{15}$

Some studies have shown cardiovascular effects of the different types of engineered nanoparticles. ${ }^{2,16}$ Using an isolated heart preparation, Stampfl et $\mathrm{al}^{16}$ demonstrated that a significant increase in heart rate accompanied by arrhythmias was evoked by three different engineered nanoparticles (flame soot, spark discharge-generated soot, and silicon dioxide). Curiously, the same authors had found no effect (heart rate, arrhythmia, or size-related effect) on flame-derived silicon dioxide nanoparticles (Aerosil ${ }^{\circledR}$; Evonik, Essen, Germany), probably due to distinct hydrophobicity or surface reactivity properties. ${ }^{17}$ Furthermore, pulmonary instillation of carbon nanoparticles exacerbated cardiac ischemia/reperfusion injury and depressed coronary flow. ${ }^{18}$ On the other hand, prolonged in vitro or in vivo exposure to carbon nanoparticles or titanium dioxide nanoparticles did not affect vasomotor responses of isolated small intrapulmonary arteries, but acute exposure to carbon nanoparticles decreased the contraction induced by vasoactive agents ${ }^{2}$ and titanium dioxide nanoparticle inhalation reduced arteriolar dilation in response to active hyperemia. ${ }^{19}$ However, few studies have addressed the effects of the MNPs on the heart and vessels.

Thus, the objectives of this study were to characterize and determine the effects of three different surface-coated manganese ferrite-based magnetic nanoparticles (citrate-coated [CiMNPs], tripolyphosphate-coated [PhMNPs], and bare magnetic nanoparticles [BaMNPs]) on: A) ventricular contractility and coronary artery vasomotricity of isolated rat hearts; B) vascular reactivity of isolated rat thoracic aortas; and C) arterial blood pressure and heart rate in unanesthetized rats.

Manganese ferrite-based nanoparticles were chosen because of their relevant magnetic resonance imaging characteristics $^{20}$ and their potential for magnetic nanoparticle hyperthermia treatment. ${ }^{21-25}$ Three nanoparticle samples were evaluated at concentrations within the range widely used in clinic $^{26,27}$ and experimental studies. ${ }^{16,28}$ Some nanoparticles were surface-coated with citrate (the coating agent already used for magnetic resonance image - see Sosnovik et $\mathrm{al}^{29}$ ) or tripolyphosphate. ${ }^{30}$ These nanoparticles (CiMNPs and PhMNPs) are stable at physiological conditions and have a negative surface charge. The third nanoparticle type (BaMNPs) is ionic and has a positive surface charge at acidic conditions; however, at physiological conditions, it is not stable and has no surface charge. As a consequence, it was named a bare nanoparticle. The negative surface-charge particles are expected to have a lower nanoparticle internalization process than positive coated (or bare) nanoparticles. ${ }^{31}$ Therefore, the idea was to evaluate the cardiovascular effects of these three nanosystems.

\section{Methods}

\section{Animals}

Male Wistar rats weighing 250-350 g were provided by the animal facilities of the Federal University of Goiás. All animals were kept in temperature-controlled rooms with 12 hour light/dark cycles and the animals had free access to water and food. All animal procedures were performed in accordance with institutional guidelines approved by local authorities.

\section{Preparation of the MNPs}

For the synthesis of manganese ferrite nanoparticles, $50 \mathrm{mmol}$ of $\mathrm{FeCl}_{3}$ and $25 \mathrm{mmol}$ of $\mathrm{MnCl}_{2}$ (both dissolved in $100 \mathrm{~mL}$ of $\mathrm{HCl} 3 \%$, weight of solute to weight of solution [w/w]) were introduced into $500 \mathrm{~mL}$ of boiling $2.0 \mathrm{~mol} / \mathrm{L}$ methylamine solution under vigorous stirring. ${ }^{32}$ After 30 minutes of reaction, the obtained solid was magnetically separated from the supernatant and washed three times with distilled water. The precipitate was acidified with a $0.5 \mathrm{~mol} / \mathrm{L} \mathrm{HNO}_{3}$ solution and magnetically separated from the supernatant, which was discarded. Thus, nanoparticles were hydrothermally treated by boiling $0.5 \mathrm{~mol} / \mathrm{L} \mathrm{Fe}\left(\mathrm{NO}_{3}\right)_{3}$ for 30 minutes and the excess ferric nitrate was removed from the solution by magnetic decantation. ${ }^{33}$ In the magnetic fluid preparation, precipitate was fractioned and nanoparticles peptized in aqueous solution, after their surface modification, using three distinct procedures, which are schematized in Figure 1: 1) for one of the samples, the precipitate was acidified with $50 \mathrm{~mL}$ of $1.0 \mathrm{~mol} / \mathrm{L} \mathrm{HNO}_{3}$ solution; 2 ) another sample was treated with sodium citrate, under stirring for 30 minutes, using a mass ratio of 1:20, $\mathrm{Na}_{3} \mathrm{C}_{6} \mathrm{H}_{5} \mathrm{O}_{7}$ to manganese ferrite, in $50 \mathrm{~mL}$ of water; and 3) in the sample, the precipitate was treated with sodium tripolyphosphate, under stirring for 60 minutes, using a mass ratio of 3:20, $\mathrm{Na}_{5} \mathrm{P}_{3} \mathrm{O}_{10}$ to manganese ferrite, in $50 \mathrm{~mL}$ of water. ${ }^{30}$ In all the cases, the obtained precipitates were magnetically separated and supernatants discarded. Afterwards, the precipitates were washed with acetone three times, then the desired amount of water was added and the excess of acetone evaporated in order to form the magnetic fluid samples. A schematic representation of the nanoparticles is presented in Figure 1. In the acidic condition, the surface sites of the oxide are protonated and the nanoparticles are positively charged. But, when diluted at physiological conditions, the nanoparticles lose their charge and become BaMNPs. On the other hand, adsorption of PhMNP 


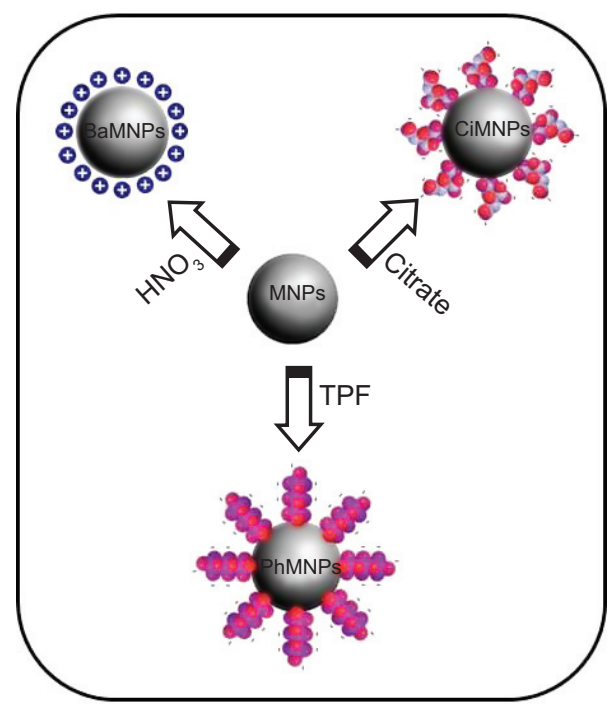

Figure I Schematic of the nanoparticle surface modification for peptization in an aqueous solution.

Notes: Manganese ferrite nanoparticles were obtained by coprecipitation. BaMNPs represents nanoparticles modified by the protonation of metal oxide surface sites with nitric acid. PhMNPs and CiMNPs represent nanoparticles that are negatively-charged and surface-modified by the adsorption of TPF and sodium citrate, respectively. Abbreviations: BaMNPs, bare magnetic nanoparticles; CiMNPs, citrate-coated magnetic nanoparticles; MNPs, magnetic nanoparticles; PhMNPs, tripolyphosphatecoated magnetic nanoparticles; TPF, sodium tripolyphosphate.

and CiMNP molecules modifies the surface of nanoparticles such that they become negatively charged due to the presence of phosphate and carboxylate anions, respectively, at the interface of solid-solution. Washing procedure reduces the ionic strength of solution and, for instance, allows for obtainment of stable colloidal sols. BaMNPs are stable at acidic conditions, while PhMNPs and CiMNPs are stable at physiological conditions. Note that the samples were synthesized at different days and do not correspond to the same batch.

\section{Characterization of the MNPs}

Magnetic characterization was obtained using a vibrating sample magnetometer model EV9 (MicroSense, East Lowell, MA, USA) with magnetic fields up to $2 \mathrm{~T}$. Both powder and colloidal samples were investigated. The magnetization data were used to extract the particle concentration of the samples. The X-ray diffraction patterns of the powders were obtained in a Shimadzu 6000 diffractometer (Shimadzu Corporation, Kyoto, Japan) using $\mathrm{Cu}\left(\mathrm{K}_{\alpha}\right)$ radiation and a $10^{\circ}$ to $80^{\circ}$ angle range. From this data, the spinel structure was confirmed. Diameter was also estimated using the Scherrer relation. Dynamic light scattering measurements of the magnetic colloids were performed using a Malvern Zetasizer Nano series (Malvern Instruments, Malvern, UK). From this data, we obtained the hydrodynamic radius of the colloidal samples and the degree of dispersion.
The magnetization characterization of the samples revealed that all of them showed a superparamagnetic behavior, ie, no hysteresis at room temperature. Figure $2 \mathrm{~A}$ shows a typical magnetization curve for the PhMNPs (powder sample). In the inset, the X-ray diffraction pattern for the sample nanoparticles is shown. The Scherrer relation was used to estimate the crystallite size (core diameter). In all the samples, the spinel structure was confirmed. Transmission electron microscopy pictures of the PhMNPs sample were obtained using a JEOL JEM-2100 (Tokyo, Japan) operating at $200 \mathrm{kV}$ (resolution $2.5 \AA$ ). The inset of Figure 2B shows a typical figure of CiMNPs. From this, a histogram (using Sturges criteria) of the size distribution of the sample was obtained. The solid line in the figure corresponds to the best fit of the data using the lognormal size distribution function:

$$
\boldsymbol{g}(\boldsymbol{D})=\left(\frac{1}{\sqrt{2 \pi} \boldsymbol{D} \delta_{D}}\right) \exp \left(-\frac{\boldsymbol{I n}^{2}\left(\frac{\boldsymbol{D}}{\boldsymbol{D}_{0}}\right)}{2 \delta_{\boldsymbol{D}}^{2}}\right)
$$

with $D, D_{0}$ and $\delta_{\mathrm{D}}$ the diameter, the median diameter, and the size dispersion, respectively. In the inset of Figure 2B, we show the median \pm standard deviation. Standard deviation (SD) was calculated from the fitting parameters of the lognormal size distribution:

$$
\mathrm{SD}=\sqrt{\left(\exp \left(\delta_{D}^{2}\right)-1\right) D_{0}^{2} \exp \left(\delta_{D}^{2}\right)}
$$

Note that the samples analyzed in this work are expected to have a broad size distribution.

Dynamic light-scattering data for all the nanofluids are presented in Figure 2C. Note that these data were performed with the original liquid carriers. So, although the ionic-based nanofluid shows a lower degree of aggregation at acidic conditions, at the cardiovascular experimental solution, one expects higher agglomeration for this sample in comparison to the others, as at the physiological $\mathrm{pH}$ there is no charge at the nanoparticle surface. Figure 2D shows the zeta potential $\mathrm{pH}$ dependence of the citrate-coated Mn-ferrite nanofluid. As expected, at the higher $\mathrm{pH}$ limit, a saturation of the zeta potential is achieved. For this sample, at physiological conditions, the zeta potential was found to be around $-40 \mathrm{mV}$, indicating a negative charge around the particle's surface, due to the carboxylic groups. Table 1 summarizes all the relevant characterization information of the samples used in this study. The magnetic particle concentration and hydrodynamic radius correspond to data of the original colloidal samples. 

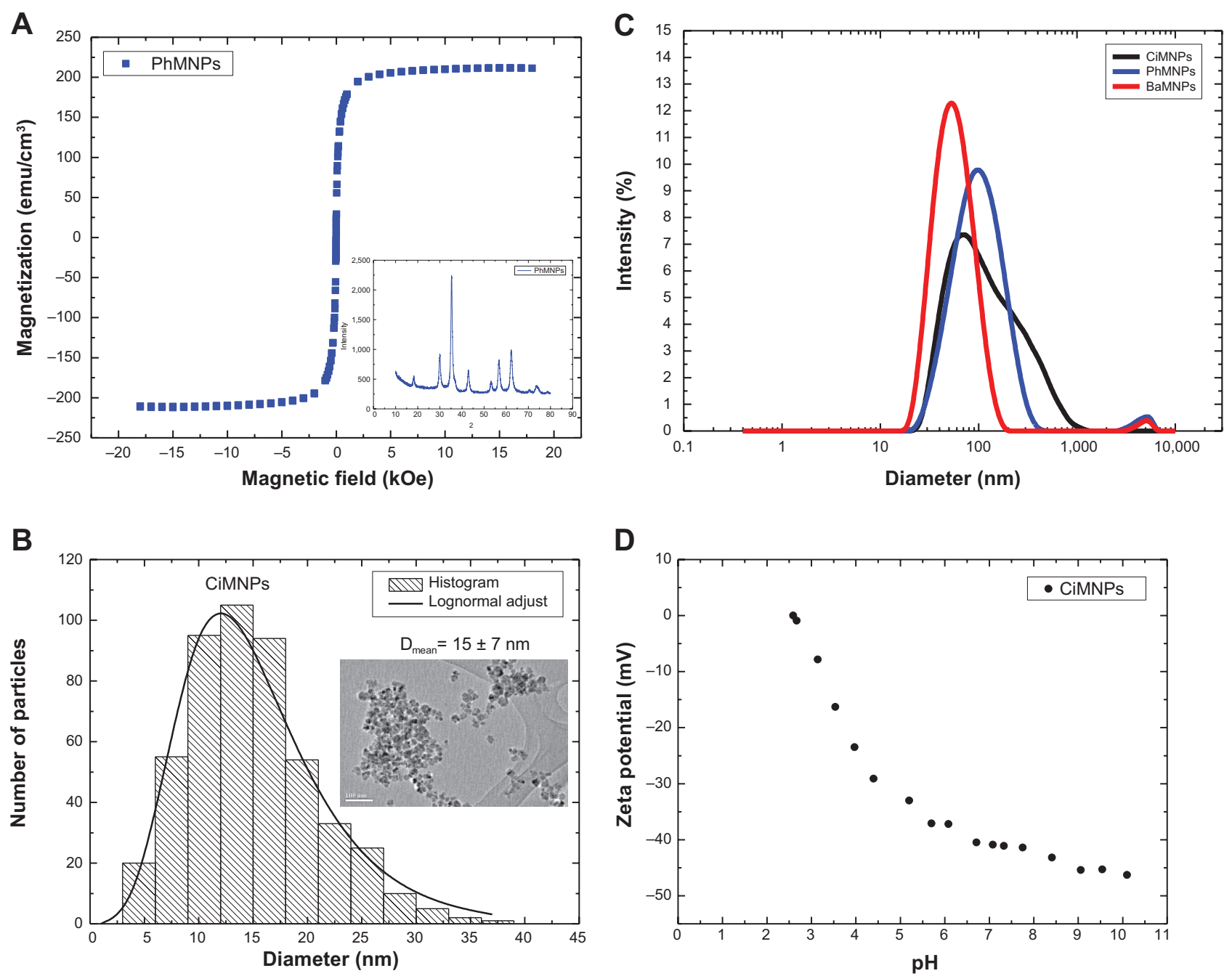

Figure 2 Characterization of the magnetic nanoparticles.

Notes: (A) Magnetization curve of manganese ferrite-based PhMNPs. The insert shows the X-ray diffraction pattern of ferrite-based nanoparticles. (B) Size distribution obtained from the transmission electron microscopy picture. The inset shows a transmission electron microscopy picture of the manganese ferrite-based CiMNPs sample. (C) Dynamic light scattering intensity as a function of hydrodynamic particle size for distinct manganese ferrite-based colloids. The BaMNPs correspond to the ionic magnetic fluid (ie, under acidic conditions) while, for the other two colloids, the samples were under physiological conditions. (D) Zeta potential pH dependence for the CiMNPsbased nanofluid.

Abbreviations: BaMNPs, bare magnetic nanoparticles; CiMNPs, citrate-coated magnetic nanoparticles; PhMNPs, tripolyphosphate-coated magnetic nanoparticles.

\section{Isolated rat hearts}

The rats were decapitated $10-15$ minutes after an intraperitoneal injection of $200 \mathrm{IU}$ heparin. The thorax was opened and the heart was carefully dissected and perfused through the aortic stump with Krebs-Ringer solution containing $\mathrm{NaCl}$ (118.4 mmol/L), $\mathrm{KCl}(4.7 \mathrm{mmol} / \mathrm{L}), \mathrm{KH}_{2} \mathrm{PO}_{4}(1.2 \mathrm{mmol} / \mathrm{L})$, $\mathrm{MgSO}_{4} \cdot 7 \mathrm{H}_{2} \mathrm{O}(1.2 \mathrm{mmol} / \mathrm{L}), \mathrm{CaCl}_{2} \cdot 2 \mathrm{H}_{2} \mathrm{O}(1.25 \mathrm{mmol} / \mathrm{L})$, glucose $(11.7 \mathrm{mmol} / \mathrm{L})$, and $\mathrm{NaHCO}_{3}(26.5 \mathrm{mmol} / \mathrm{L})$.

Table I Sample parameters obtained from X-ray diffraction, VSM, and dynamic light scattering characterization techniques

\begin{tabular}{lllllll}
\hline Sample & Coating layer & $\begin{array}{l}\text { Core diameter } \\
(\mathbf{n m})\end{array}$ & $\begin{array}{l}\text { Hydrodynamic } \\
\text { diameter } \mathbf{( n m})\end{array}$ & $\begin{array}{l}\text { Particle magnetization } \\
\left(\mathbf{e m u} / \mathbf{c m}^{3}\right)\end{array}$ & $\begin{array}{l}\text { Zeta potential } \\
(\mathbf{m V})\end{array}$ & $\begin{array}{l}\text { Particle concentration } \\
(\mathbf{m g} / \mathbf{m L})\end{array}$ \\
\hline CiMNPs & Citrate & 14.0 & 90.2 & 167 & -42 & 106.5 \\
PhMNPs & Phosphate & 13.1 & 87.9 & 236 & -41 & 29.5 \\
BaMNPs & None & 9.2 & 51.8 & 270 & None & 50.0 \\
\hline
\end{tabular}

Notes: The dynamic light scattering data and particle concentrations correspond to the original magnetic colloidal samples. Zeta potential corresponds to data under physiological conditions.

Abbreviations: BaMNPs, bare magnetic nanoparticles; CiMNPs, citrate-coated magnetic nanoparticles; PhMNPs, tripolyphosphate-coated magnetic nanoparticles; VSM, vibrating sample magnetometer. 
The perfusion flow was maintained at a constant rate $(10 \mathrm{~mL} / \mathrm{min})$ at $37^{\circ} \mathrm{C}$ and with constant oxygenation $\left(5 \% \mathrm{CO}_{2}\right.$ and $\left.95 \% \mathrm{O}_{2}\right)$. A balloon was inserted into the left ventricle through the left atrium for isovolumetric recordings of left ventricular pressures. Coronary perfusion pressure was measured with a pressure transducer that was connected to the aortic cannula. Data were analyzed online using a data acquisition system (DATAQ Instruments, Akron, OH, USA). Hearts having left ventricular end-systolic pressure less than $60 \mathrm{mmHg}$, heart rate less than $200 \mathrm{bpm}$, and perfusion pressure less than $60 \mathrm{mmHg}$ and higher than $140 \mathrm{mmHg}$ at the end of the stabilization period were excluded. After a basal period (30-40 minutes), the hearts were perfused for an additional 10 minutes with Krebs-Ringersolution-containing MNPs at the mean concentration of the $0.6 \mu \mathrm{g} / \mathrm{mL}$. Thereafter, the hearts were perfused with MNPfree solution for 20 minutes (the washout period).

\section{Isolated aortic ring preparation}

Isolated aortic rings were used to evaluate the effects of MNPs in rat thoracic aortas. Aortic rings $(4 \mathrm{~mm})$ from descending thoracic aortas were placed in $10 \mathrm{~mL}$ organ baths at $37^{\circ} \mathrm{C}$ containing gassed $\left(95 \% \mathrm{O}_{2}\right.$ and $5 \%$ $\mathrm{CO}_{2}$ ) Krebs-Henseleit solution with the following composition: $\mathrm{NaCl}(118.06 \mathrm{mmol} / \mathrm{L}), \mathrm{KCl}(4.6 \mathrm{mmol} / \mathrm{L})$, $\mathrm{NaHCO}_{3}(24.9 \mathrm{mmol} / \mathrm{L}), \mathrm{MgSO}_{4} \cdot 7 \mathrm{H}_{2} \mathrm{O}(2.4 \mathrm{mmol} / \mathrm{L})$, $\mathrm{CaCl}_{2} \cdot 2 \mathrm{H}_{2} \mathrm{O}(3.3 \mathrm{mmol} / \mathrm{L}), \mathrm{KH}_{2} \mathrm{PO}_{4}(0.9 \mathrm{mmol} / \mathrm{L})$, and glucose $(11.1 \mathrm{mmol} / \mathrm{L})$. The rings were maintained under a tension of $1.5 \mathrm{~g}$ for 1 hour to equilibrate. Mechanical activity was recorded isometrically using a data acquisition system (DATAQ Instruments). To evaluate the possible constriction effects of MNPs, the aortic rings were incubated with increasing concentrations of MNPs in the basal condition. The relaxation effect of the MNPs was assayed in vessels preconstricted with phenylephrine $(0.1 \mu \mathrm{mol} / \mathrm{L})$. For each protocol, 6.2, 31.0, 62.0, 310.0, and $620.0 \mu \mathrm{g} / \mu \mathrm{L}$ of MNPs were added to the Krebs-Henseleit solution in order.

\section{Blood pressure measurement}

The rats were anesthetized (using ketamine $70 \mathrm{mg} \cdot \mathrm{kg}^{-1}$ and xylazine $30 \mathrm{mg} \cdot \mathrm{kg}^{-1}$ ), and polyethylene catheters (PE-10) were inserted into the right femoral artery and vein, tunneled under the skin, and exteriorized at the neck. Mean arterial pressure (MAP) was measured in conscious rats 24 hours after recovery from anesthesia. MNPs were infused intravenously at graded doses $(0.41,0.82,1.64,3.28$, and $6.56 \mathrm{mg} / \mathrm{kg})$ after an initial control recording for 30 minutes. Doses were administered at intervals of approximately 15 minutes. To evaluate the longterm effect of MNPs, recordings of MAP were obtained 24 hours after obtaining a curve over a 30-minute period (preceded by 30 minutes of equilibration). The data were recorded on a data acquisition system (DATAQ Instruments).

\section{Data analysis}

The results are presented as the mean \pm standard error. A two-way analysis of variance with a Fisher's least significant difference multiple comparison post-test was used to compare the curves obtained from the preparation of isolated hearts and aortic rings. A one-way analysis of variance followed by the Newman-Keuls post-test was used to analyze the MAP and heart rate. All statistical analyses were considered significant at $P<0.05$.

\section{Results}

To evaluate the direct effects of the MNPs on cardiac contractility, isolated rat hearts were perfused with MNPs (BaMNPs, CiMNPs, and PhMNPs). As observed in Figure 3A, CiMNPs induced a transient decrease in the left ventricular end-systolic pressure (LVESP). This effect was not observed in PhMNPs or BaMNPs (Figure 3B and C). On the other hand, PhMNPs and BaMNPs, but not CiMNPs, induced a significant increase in the left ventricular enddiastolic pressure (LVEDP) (Figure 4), which resulted in a decrease in the left ventricular developed pressure (LVDevP; calculated as the difference between LVESP and LVEDP) (Figure 5). These effects were not reversed during the washout period. Similarly, PhMNPs and BaMNPs produced a decrease in the maximal rate of left ventricular pressure rise $(+\mathrm{dP} / \mathrm{dt})$ (Figure 6$)$ and maximal rate of left ventricular pressure decline $(-\mathrm{dP} / \mathrm{dt})$ (Figure 7$)$ at the end of the infusion of the fluids. These effects were not reversed during the washout period. The heart rate of the isolated hearts was reduced by the infusion of the CiMNPs and BaMNPs (Figure 8A and C). The PhMNPs did not affect heart rate (Figure $8 \mathrm{~B}$ ).

We also evaluated the effects of the MNPs on coronary vasomotricity. The CiMNPs and PhMNPs did not change the perfusion pressure during infusion of the fluids, although both induced an increase in perfusion pressure during the washout period (Figure 9A and B). In contrast, the perfusion pressure was increased at the beginning of the infusion of the BaMNPs and it remained higher during the washout period (Figure 9C).

The effects of the MNPs on the systemic vasculature (aortic rings) were also evaluated. As observed in Figure 10A, none of the MNPs analyzed produced a constrictor effect. In a second set of experiments, the aorta rings were preconstricted 

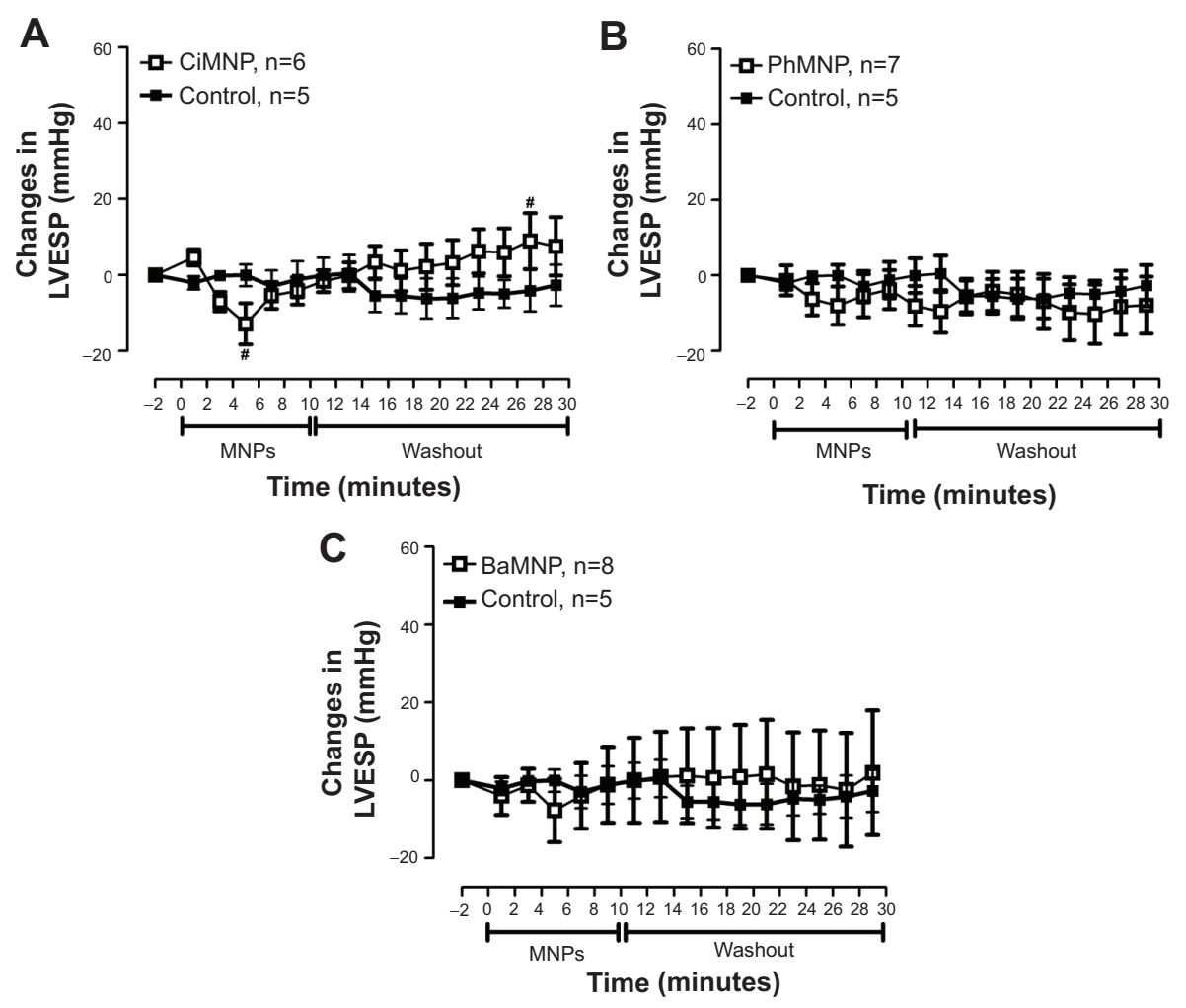

Figure 3 The effects of CiMNP, PhMNP, and BaMNP on left ventricular end-systolic pressure in isolated perfused hearts.

Notes: (A) CiMNP, (B) PhMNP, and (C) BaMNP. After a basal period, the hearts were perfused for 10 minutes with a Krebs-Ringer solution containing magnetic nanoparticles followed by a 20 -minute washout period. ${ }^{\sharp} P<0.01$ between time points. Values are shown as the mean \pm standard error. A two-way analysis of variance followed by a post-hoc Fisher's least significant difference test was used.

Abbreviations: BaMNP, bare magnetic nanoparticles; CiMNP, citrate-coated magnetic nanoparticles; LVESP, left ventricular end-systolic pressure; MNPs, magnetic nanoparticles; PhMNP, tripolyphosphate-coated magnetic nanoparticles.
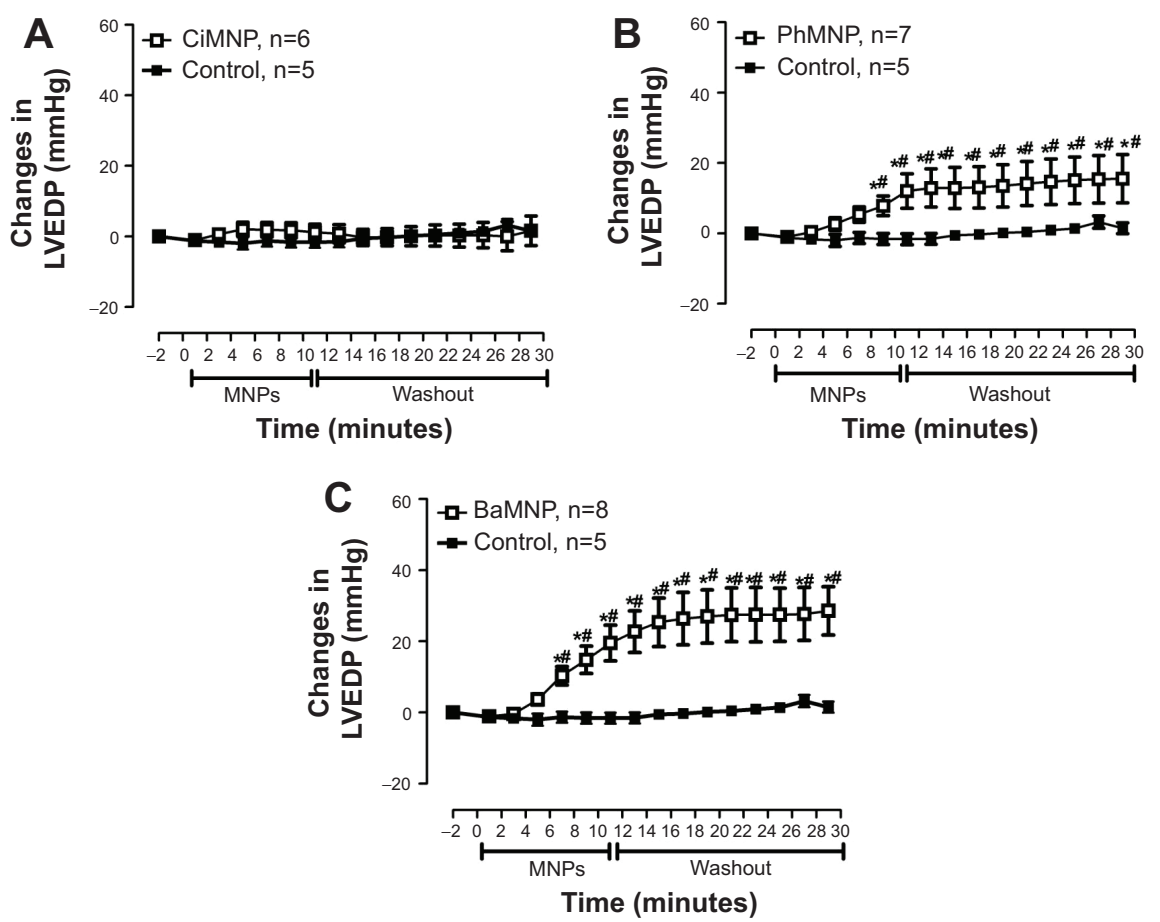

Figure 4 The effects of $\mathrm{CiMNP}, \mathrm{PhMNP}$, and BaMNP on left ventricular end-diastolic pressure in isolated perfused hearts.

Notes: (A) CiMNP, (B) PhMNP, and (C) BaMNP. After a basal period, the hearts were perfused for 10 minutes with a Krebs-Ringer solution containing magnetic nanoparticles followed by a 20 -minute washout period. ${ }^{*} P<0.0$ I versus basal level, ${ }^{*} P<0.01$ between time points. Values are shown as the mean \pm standard error. $A$ two-way analysis of variance followed by a post-hoc Fisher's least significant difference test was used.

Abbreviations: BaMNP, bare magnetic nanoparticles; CiMNP, citrate-coated magnetic nanoparticles; LVEDP, left ventricular end-diastolic pressure; MNPs, magnetic nanoparticles; PhMNP, tripolyphosphate-coated magnetic nanoparticles. 

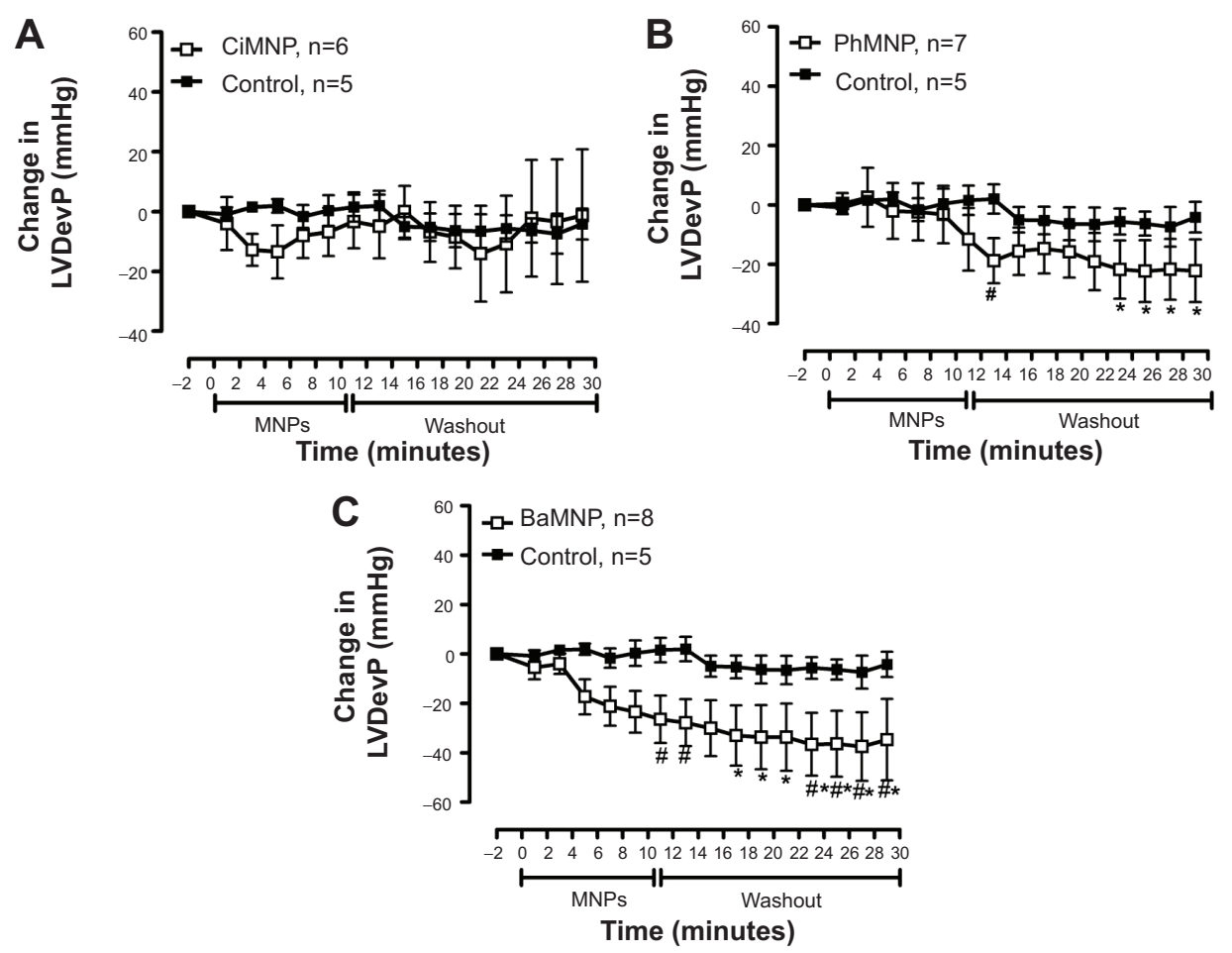

Figure 5 The effects of CiMNP, PhMNP, and BaMNP on left ventricular developed pressure in isolated perfused hearts.

Notes: (A) CiMNP, (B) PhMNP, and (C) BaMNP. After a basal period, the hearts were perfused for 10 minutes with a Krebs-Ringer solution containing magnetic nanoparticles followed by a 20 -minute washout period. ${ }^{*} P<0.01$ versus basal level, ${ }^{*} P<0.01$ between time points. Values are shown as the mean \pm standard error. $A$ two-way analysis of variance followed by a post-hoc Fisher's least significant difference test was used.

Abbreviations: BaMNP, bare magnetic nanoparticles; CiMNP, citrate-coated magnetic nanoparticles; LVDevP, left ventricular developed pressure; MNPs, magnetic nanoparticles; PhMNP, tripolyphosphate-coated magnetic nanoparticles.
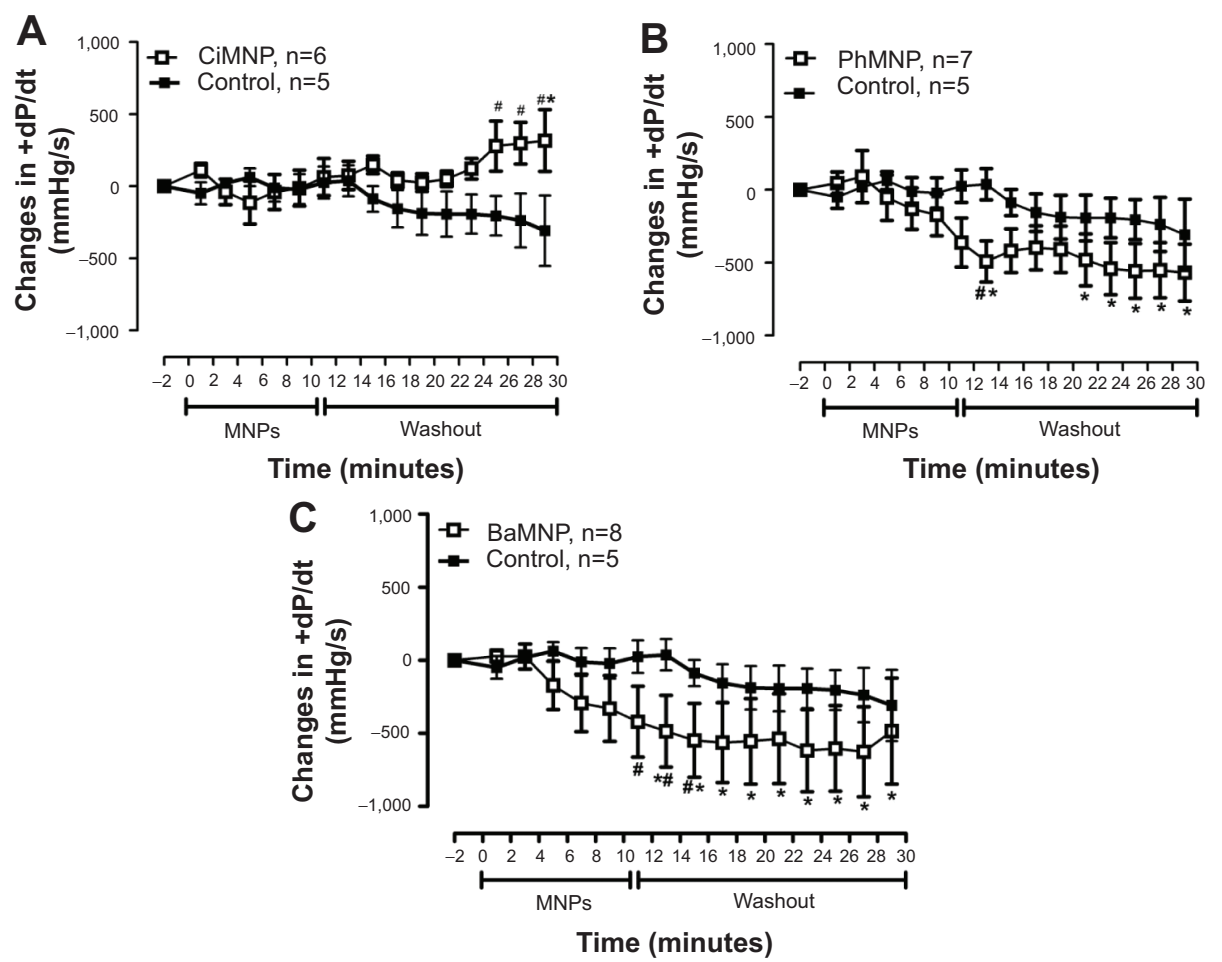

Figure 6 The effects of CiMNP, PhMNP, and BaMNP on $+\mathrm{dP} / \mathrm{dt}$ in isolated perfused hearts.

Notes: (A) CiMNP, (B) PhMNP, and (C) BaMNP. After a basal period, the hearts were perfused for 10 minutes with a Krebs-Ringer solution containing magnetic nanoparticles followed by a 20 -minute washout period. $+\mathrm{dP} / \mathrm{dt}$, maximal rate of left ventricular pressure rise. $* P<0.0 \mathrm{I}$ versus basal level, ${ }^{\# P}<0.0 \mathrm{I}$ between time points. Values are shown as the mean \pm standard error. Two-way analysis of variance followed by post-hoc Fisher's least significant difference test.

Abbreviations: BaMNP, bare magnetic nanoparticles; CiMNP, citrate-coated magnetic nanoparticles; MNPs, magnetic nanoparticles; PhMNP, tripolyphosphate-coated magnetic nanoparticles. 

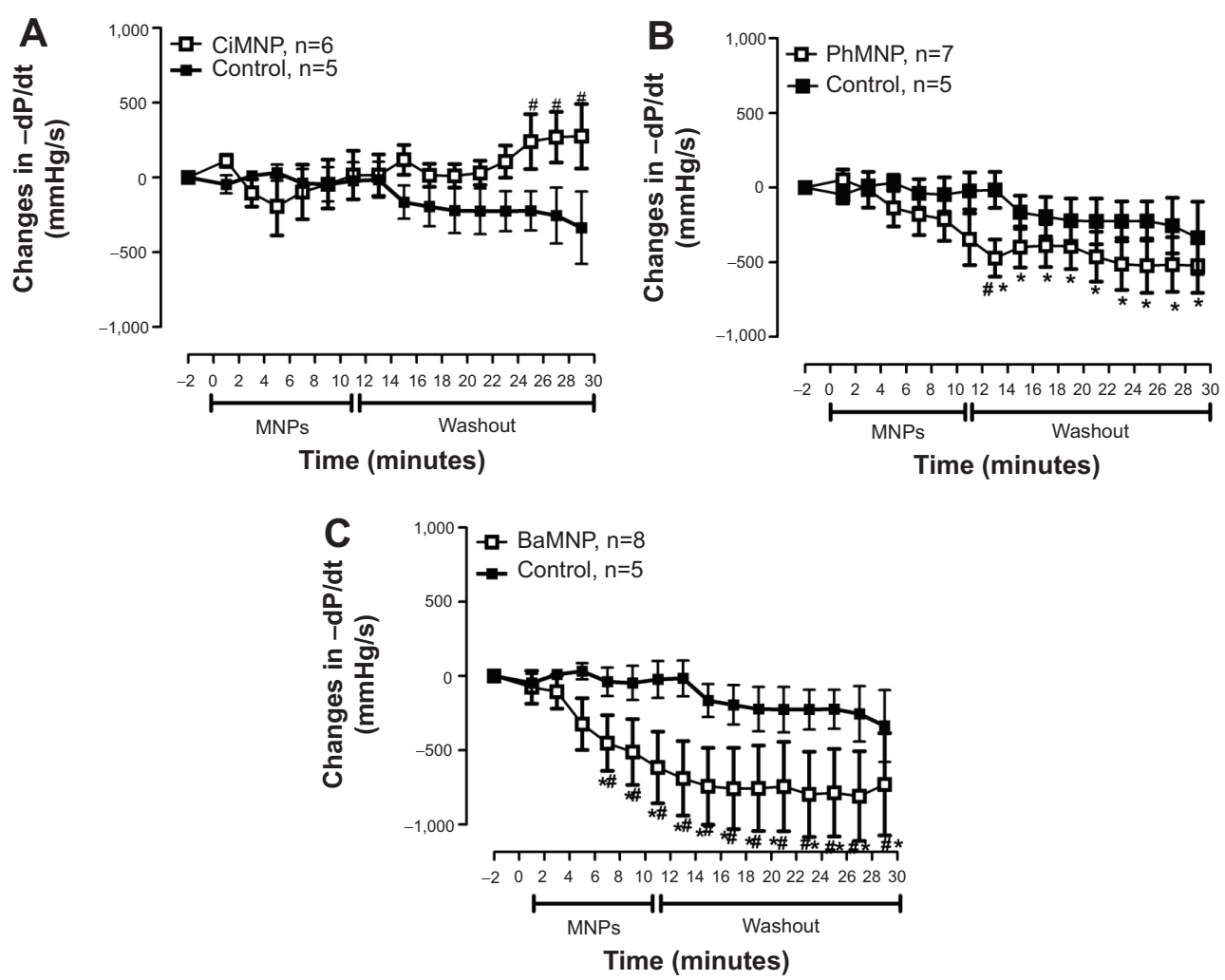

Figure 7 The effects of $\mathrm{CiMNP}, \mathrm{PhMNP}$, and $\mathrm{BaMNP}$ on -dP/dt in isolated perfused hearts.

Notes: (A) CiMNP, (B) PhMNP, and (C) BaMNP. After a basal period, the hearts were perfused for 10 minutes with Krebs-Ringer solution containing magnetic nanoparticles followed by a 20 -minute washout period. $-\mathrm{dP} / \mathrm{dt}$, maximal rate of left ventricular pressure decline. ${ }^{* P}<0.0 \mathrm{I}$ versus basal level, ${ }^{\sharp P}<0.0 \mathrm{I}$ between time points. $V$ alues are shown as the mean \pm standard error. A two-way analysis of variance followed by a post-hoc Fisher's least significant difference test was used.

Abbreviations: BaMNP, bare magnetic nanoparticles; CiMNP, citrate-coated magnetic nanoparticles; MNPs, magnetic nanoparticles; PhMNP, tripolyphosphate-coated magnetic nanoparticles.
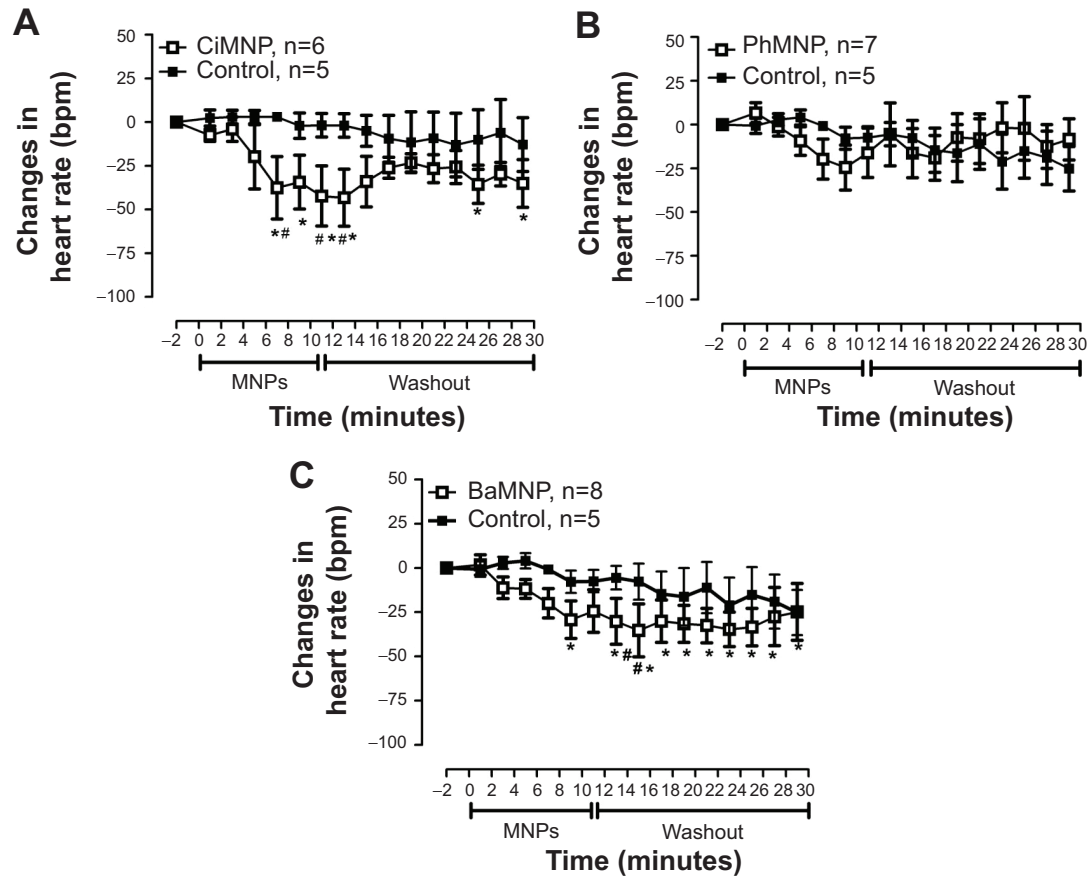

Figure 8 The effects of CiMNP, PhMNP, and BaMNP on heart rate in isolated perfused hearts.

Notes: (A) CiMNP, (B) PhMNP, and (C) BaMNP. After a basal period, the hearts were perfused for 10 minutes with a Krebs-Ringer solution containing magnetic nanoparticles followed by a 20 -minute washout period. ${ }^{*} P<0.01$ versus basal level, ${ }^{*} P<0.01$ between time points. Values are shown as the mean \pm standard error. $A$ two-way analysis of variance followed by a post-hoc Fisher's least significant difference test was used.

Abbreviations: BaMNP, bare magnetic nanoparticles; CiMNP, citrate-coated magnetic nanoparticles; MNPs, magnetic nanoparticles; PhMNP, tripolyphosphate-coated magnetic nanoparticles. 


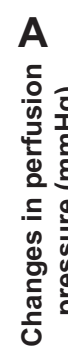

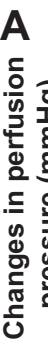
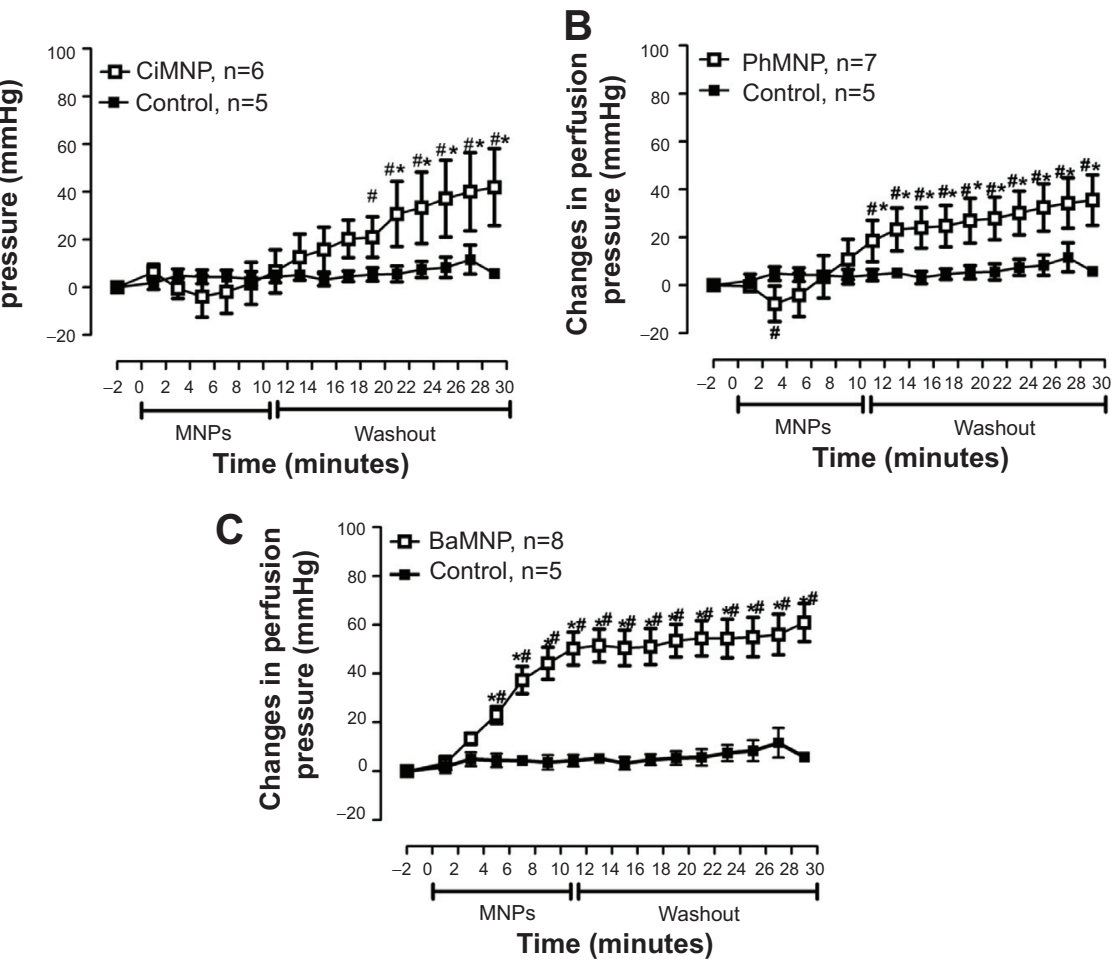

Figure 9 The effects of CiMNP, PhMNP, and BaMNP on perfusion pressure in isolated perfused hearts.

Notes: (A) CiMNP, (B) PhMNP, and (C) BaMNP. After a basal period, the hearts were perfused for 10 minutes with a Krebs-Ringer solution containing magnetic nanoparticles followed by a 20 -minute washout period. ${ }^{*} P<0.0$ I versus basal level, ${ }^{\#} P<0.0$ l between time points. Values are shown as the mean \pm standard error. A two-way analysis of variance followed by a post-hoc Fisher's least significant difference test was used.

Abbreviations: BaMNP, bare magnetic nanoparticles; CiMNP, citrate-coated magnetic nanoparticles; MNPs, magnetic nanoparticles; PhMNP, tripolyphosphate-coated magnetic nanoparticles.
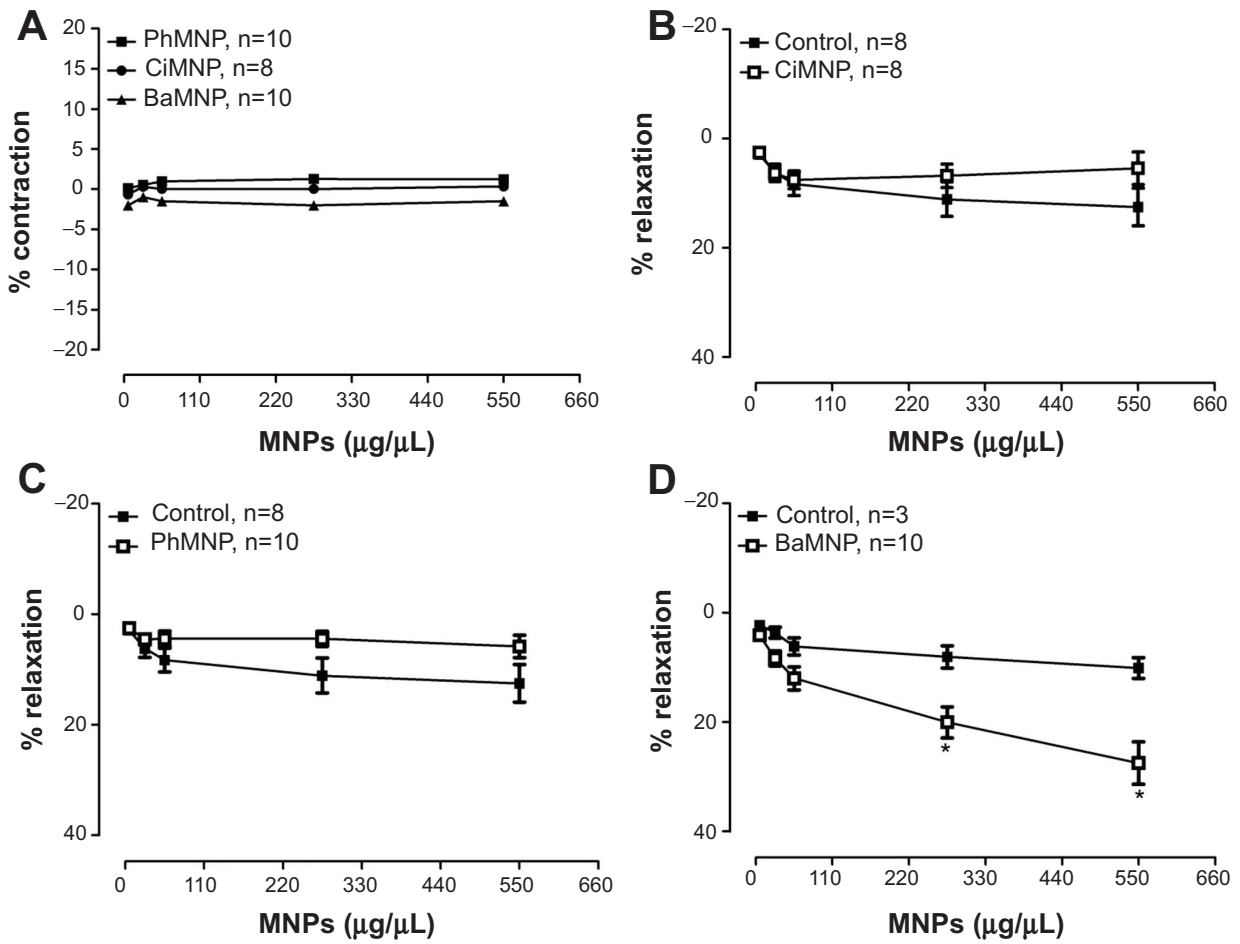

Figure 10 Effects of MNPs on the isolated aortic rings of rats.

Notes: (A) Cumulative concentration-response curves of the MNPs in aortic rings with intact endothelium without preconstriction. The effects of (B) CiMNP, (C) PhMNP, and (D) BaMNP on the vasorelaxant response in the aortic rings with intact endothelium preconstricted by phenylephrine $(0.1 \mu \mathrm{M})$. Each dose was administered at intervals of approximately I minute. ${ }^{*} \mathrm{P}<0.0 \mathrm{I}$. Values are shown as the mean \pm standard error. A two-way analysis of variance followed by a post-hoc Fisher's least significant difference test was used.

Abbreviations: BaMNP, bare magnetic nanoparticles; CiMNP, citrate-coated magnetic nanoparticles; MNPs, magnetic nanoparticles; PhMNP, tripolyphosphate-coated magnetic nanoparticles. 
and the fluids were added to assess the relaxation effects. The BaMNPs, but not CiMNPs or PhMNPs, presented a significant vasorelaxant effect (Figure 10B-D).

In contrast to the ex vivo effects, MNPs were not able to induce any cardiovascular effects in conscious rats. Basal values of the MAP and heart rate are presented in Table S1. Intravenous injections of the fluids did not induce acute changes in blood pressure (Figure 11A-C) or heart rate (Figure 11D-F). In addition, the measurements were also performed 24 hours after the injections and no changes were observed (Figures 11A-F, insets).

\section{Discussion}

The major findings of this paper were that the MNPs (bare or coated) produced by the coprecipitation method induced direct effects on the ventricular contractility and coronary vasomotricity in isolated hearts. These effects were strongly dependent upon the surface-coating layer of the nanoparticles.
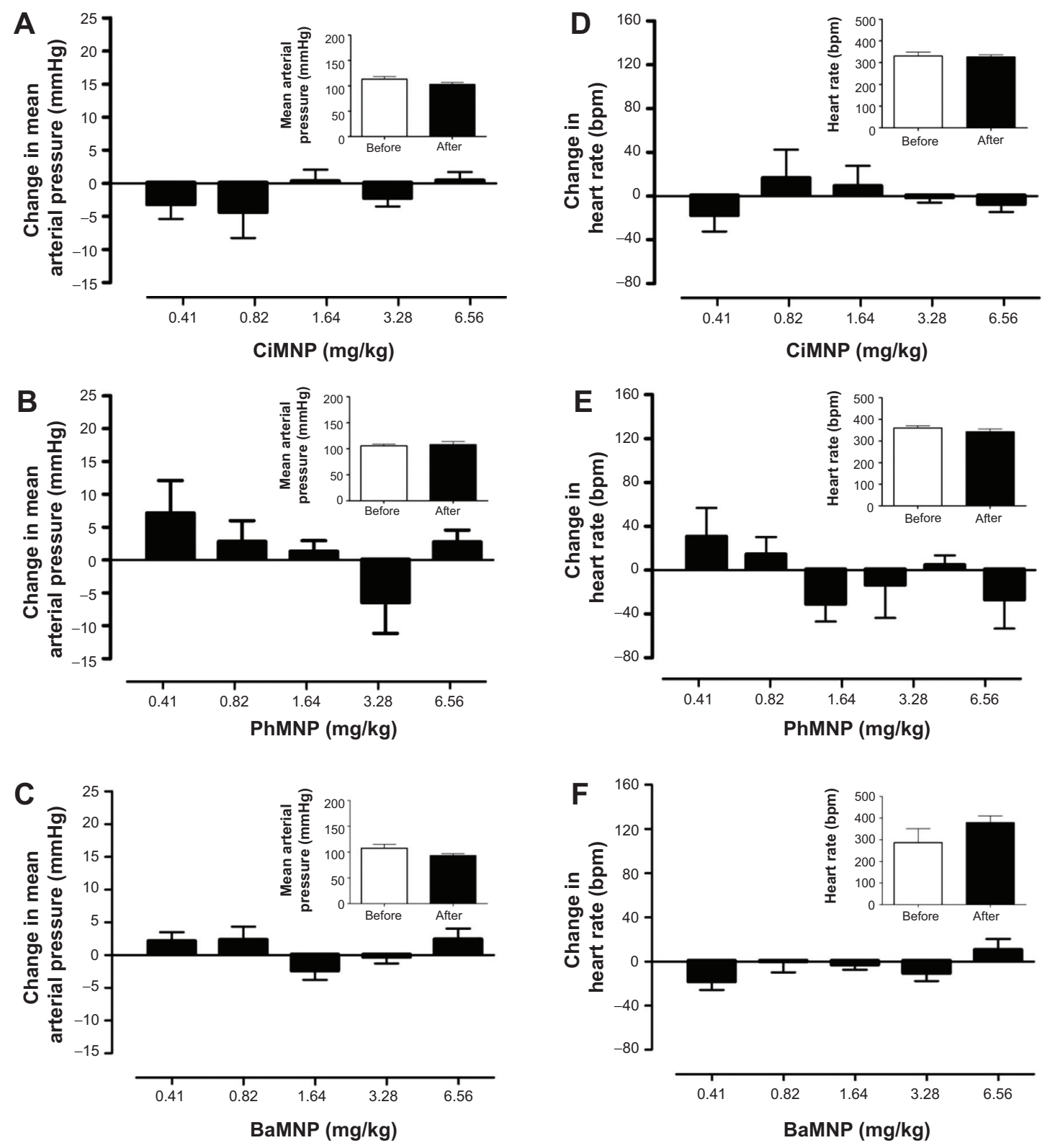

Figure II The effects of CiMNP, PhMNP, and BaMNP on mean arterial pressure and heart rate in conscious rats.

Notes: Effect of $(\mathbf{A})$ CiMNP, (B) PhMNP, and (C) BaMNP on mean arterial pressure. Effect of (D) CiMNP, (E) PhMNP, and (F) BaMNP on heart rate. The magnetic nanoparticles were infused intravenously at graded doses. Doses were administered at intervals of approximately 15 minutes. Values are shown as the mean \pm standard error. A one-way analysis of variance was followed by a Newman-Keuls posttest. The insets show the mean arterial pressure or heart rate obtained 24 hours after the graded doses of the magnetic nanoparticles. Bars represent the average of three values (values were collected at 5-minute intervals after the basal period). Values are shown as the mean \pm standard error and were determined by a Student's $t$-test $(n=4-5)$.

Abbreviations: BaMNP, bare magnetics nanoparticles; CiMNP, citrate-coated magnetic nanoparticles; PhMNP, tripolyphosphate-coated magnetic nanoparticles. 
On the other hand, we did not observed any effects on heart rate or arterial blood pressure in conscious rats

The data of the isolated hearts are consistent with previous studies that show that MNPs induce cardiovascular effects. ${ }^{28}$ Here, we have used three different MNPs that each induced similar effects. It is likely that MNPs are able to cross the vascular endothelial monolayer ${ }^{34}$ and induce direct effects on cardiomyocytes. These effects can be triggered by internalization of the MNPs in the cardiomyocytes, and this phenomenon has been observed in different types of cells. ${ }^{35-37}$ In addition, Wu et a ${ }^{38}$ showed that particle size and surface modification lead to different responses in terms of the uptake of iron oxide nanoparticles. It is important to note that the contractile effects observed in the isolated hearts were not reversible during the washout period. This strengthens the hypothesis that the MNPs are taken up by cardiomyocytes.

The main effect observed in the isolated heart preparation was that MNPs induced an increase in LVEDP and a decrease in left ventricular developed pressure. These changes are generally related to cytosolic calcium overload in cardiomyocytes. ${ }^{39}$ Thus, we speculated that the MNPs were shifting the calcium handling in response to an increase in sarcoplasmic reticulum calcium release or increase in calcium influx across the plasma membrane. However, it is possible that the cardiac effects can be generated by $\mathrm{Mn}^{2+}$ itself since previous studies have shown that $\mathrm{Mn}^{2+}$ can enter in the excitable cells via calcium channels or transporters and bind intracellularly due its affinity for $\mathrm{Ca}^{2+}$ and $\mathrm{Mg}^{2+}$ binding sites on proteins. ${ }^{40}$ So, possibly, when inside cells, due to a different $\mathrm{pH}$ environment, some ions can be released from the nanoparticle surface. This hypothesis, however, deserves a more profound investigation and will be left for future works.

The infusion of BaMNPs induced a more pronounced effect on contractility of the isolated hearts when compared with the negative-charge-coated nanoparticles. Importantly, a decrease in particle internalization of negatively coated nanoparticles has been reported previously. ${ }^{31}$ Indeed, theoretical models indicate that repulsive nonspecific interactions (arising from the negatively charged coating layers) might be responsible for such an effect. ${ }^{41}$ Furthermore, Decuzzi and Ferrari ${ }^{42}$ developed a mathematical model that included the adhesive and endocytic properties of nanoparticles interacting with cells. In particular, they described a phase-like diagram with three regions, namely: no particle adhesion; firm adhesion with no endocytosis; and firm adhesion with endocytosis. ${ }^{42,43}$ The governing parameters were found to be geometrical (shape, size), biophysical (ligand-to-receptor surface density ratio, hydrodynamic force parameter, particle surface charge, among others), and biological (ligand-receptor binding affinity, etc). In general, the model stated that the adhesion time decreased as the particle got smaller, ie, it was easier for lower-size particles to firmly adhere to a cell surface. Therefore, these results suggest a higher affinity for the cellular membrane from the BaMNPs, and this might also help to explain the more pronounced effect of the BaMNPs. Thus, the enhanced cardiovascular effect might be related to higher adhesion in the membrane and/or endocytosis from those nanoparticles.

All three MNPs studied induced an increase in the perfusion pressure of isolated hearts. We could argue that this effect was secondary to the rise in the LVEDP, promoting a compressive effect in the coronary vasculature. However, CiMNPs evoked an increase in the perfusion pressure without changes in the intraventricular systolic or diastolic pressure, suggesting that MNPs have a direct effect in the coronary arteries.

We also evaluated the vascular effects of MNPs in isolated aortic rings. Only BaMNPs were able to induce a slight vasorelaxant effect in these vessels. To date, our study is the first to evaluate the effects of MNPs in isolated vessels. Previous studies have shown direct vascular effects of nonmagnetic nanoparticles. In isolated rat aortic rings, a low concentration of silver nanoparticles induced vasoconstriction and a high concentration stimulated nitrogen oxide-mediated vasorelaxation. ${ }^{44}$ In mesenteric arteries, the response to noradrenaline was significantly lower in arteries from mice injected with iron oxide nanoparticles, as well as in ex vivo incubated vessels. ${ }^{28}$ In addition, Courtois et $\mathrm{al}^{2}$ reported that carbon-manufactured nanoparticles altered pulmonary vascular tone, whereas titanium dioxide nanoparticles - another class of nanomaterials - did not.

Alternatively, cardiovascular effects evoked by magnetic and nonmagnetic nanoparticles have been reported in a few previous studies. ${ }^{28,34,44,45}$ Although some studies demonstrate that MNPs are relatively stable in vivo, ${ }^{46}$ it has been observed that intravenously-injected MNPs can be removed from the circulation by the reticuloendothelial system, culminating in a blood half-life of approximately 24 hours in humans and 11 hours in mice.$^{47}$ However, this half-life and the small size of the particles allows them to penetrate tissues such as the heart. ${ }^{48}$ Indeed, it is important to note that, in our study, the MNPs were infused directly into the femoral vein and, therefore, were expected to reach the heart within few seconds. Also, the concentration used in vivo was several times higher than that used in ex vivo studies. Thus, we believe that the MNPs are taken up in the vessels as well as the heart without inducing significant effects. 
We can also hypothesize that the lack of in vivo effects can be related to the ability of nanoparticles to progressively and selectively adsorb biomolecules when they come into contact with biological fluids. ${ }^{49}$ This opsonization phenomenon (also known as the corona effect ${ }^{49}$ ) was found to depend on several nanoparticle properties, namely: ${ }^{17}$ surface charge, where the rate of protein adsorption was found to be slower for neutral nanoparticles; hydrophobicity, which seems to influence the amount of proteins bound to and also the identities of the nanoparticles, while size, shape, and morphology seem to influence only the amount of bound proteins but not their identities; and surface coating, which strongly influences protein binding. For example, CiMNPs are expected to have many proteins bound to their surface, while PEGylated particles have comparatively few bound proteins. It is interesting to note that all of these parameters have a strong influence on the adhesive and endocytic properties of nanoparticles interacting with cells. Although several of these effects have already been reported, ${ }^{17,49}$ the understanding of this nanoparticle-protein interaction is still in its infancy. Therefore, other studies are necessary to better elucidate the interaction between the MNPs and serum proteins.

\section{Conclusion}

In summary, we report that MNPs induced cardiovascular effects only in isolated heart and vessel preparations. However, these particles failed to change hemodynamic parameters in conscious rats. These results indicate that it is important to carefully analyze the data obtained from isolated organs to avoid misinterpretation of the results and conclusions. Thus, given the proper dosages, we conclude that these MNPs should be considered for possible therapeutic applications.

\section{Acknowledgment}

This work was supported by grants from the Conselho Nacional de Ciência e Tecnologico (CNPQ), Coordenação de Aperfeiçoamento de Pessoal de Nível Superior (CAPES), and Fundação de Amparo à Pesquisa do Estado de Goiás (FAPEG).

\section{Disclosure}

The authors report no conflicts of interest in this work.

\section{References}

1. De Jong WH, Borm PJ. Drug delivery and nanoparticles: applications and hazards. Int J Nanomedicine. 2008;3:133-149.

2. Courtois A, Andujar P, Ladeiro Y, et al. Effect of engineered nanoparticles on vasomotor responses in rat intrapulmonary artery. Toxicol Appl Pharmacol. 2010;245:203-210.

3. Ruiz-Esparza GU, Flores-Arredondo JH, Segura-Ibarra V, et al. The physiology of cardiovascular disease and innovative liposomal platforms for therapy. Int J Nanomedicine. 2013;8:629-640.
4. Bakuzis AF, Branquinho LC, e Castro LL, e Eloi MT, Miotto R. Chain formation and aging process in biocompatible polydisperse ferrofluids: experimental investigation and Monte Carlo simulations. Adv Colloid Interface Sci. 2013;191-192:1-21.

5. Kundra SN. Toward the emergence of nanoneurosurgery: part IIInanomedicine: targeted nanotherapy, nanosurgery and progress toward the realization of nanoneurosurgery. Neurosurgery. 2008;62:E1384.

6. Ito A, Honda H, Kobayashi T. Cancer immunotherapy based on intracellular hyperthermia using magnetite nanoparticles: a novel concept of "heat-controlled necrosis" with heat shock protein expression. Cancer Immunol Immunother. 2006;55:320-328.

7. Alivisatos P. The use of nanocrystals in biological detection. Nat Biotechnol. 2004;22:47-52.

8. Lu CW, Hung Y, Hsiao JK, et al. Bifunctional magnetic silica nanoparticles for highly efficient human stem cell labeling. Nano Lett. 2007; 7:149-154.

9. Boulaiz H, Alvarez PJ, Ramirez A, et al. Nanomedicine: application areas and development prospects. Int J Mol Sci. 2011;12:3303-3321.

10. Wilczewska AZ, Niemirowicz K, Markiewicz K, Car H. Nanoparticles as drug delivery systems. Pharmacol Rep. 2012;64:1020-1037.

11. Ito A, Shinkai M, Honda H, Kobayashi T. Medical application of functionalized magnetic nanoparticles. J Biosci Bioeng. 2005;100:1-11.

12. Gupta AK, Gupta M. Synthesis and surface engineering of iron oxide nanoparticles for biomedical applications. Biomaterials. 2005;26: 3995-4021.

13. Cintra ER, Ferreira FS, Santos Junior JL, et al. Nanoparticle agglomerates in magnetoliposomes. Nanotechnology. 2009;20:045103.

14. Oliveira RR, Ferreira FS, Cintra ER, Branquinho LC, Bakuzis AF, Lima EM. Magnetic nanoparticles and rapamycin encapsulated into polymeric nanocarriers. J Biomed Nanotechnol. 2012;8:193-201.

15. McCarthy JR, Weissleder R. Multifunctional magnetic nanoparticles for targeted imaging and therapy. Adv Drug Del Rev. 2008;60: $1241-1251$.

16. Stampfl A, Maier M, Radykewicz R, Reitmeir P, Göttlicher M, Niessner R. Langendorff heart: a model system to study cardiovascular effects of engineered nanoparticles. ACS Nano. 2011;5:5345-5353.

17. Aggarwal P, Hall JB, McLeland CB, Dobrovolskaia MA, McNeil SE. Nanoparticle interaction with plasma proteins as it relates to particle biodistribution, biocompatibility and therapeutic efficacy. Adv Drug Deliv Rev. 2009;61:428-437.

18. Thompson LC, Frasier CR, Sloan RC, et al. Pulmonary instillation of multi-walled carbon nanotubes promotes coronary vasoconstriction and exacerbates injury in isolated hearts. Nanotoxicology. 2014;8(1): $38-49$.

19. Knuckles TL, Yi J, Frazer DG, et al. Nanoparticle inhalation alters systemic arteriolar vasoreactivity through sympathetic and cyclooxygenasemediated pathways. Nanotoxicology. 2012;6:724-735.

20. Kim HM, Lee H, Hong KS, et al. Synthesis and high performance of magnetofluorescent polyelectrolyte nanocomposites as MR/ Near-infrared multimodal cellular imaging. ACS Nano. 2011;5: $8230-8240$.

21. Huang H, Delikanli S, Zeng H, Ferkey DM, Pralle A. Remote control of ion channels and neurons through magnetic-field heating of nanoparticles. Nat Nanotechnol. 2010;5:602-606.

22. Jeun M, Moon SJ, Kobayashi H, et al. Effects of Mn concentration on the ac magnetically induced heating characteristics of superparamagnetic $\mathrm{Mn}_{\mathrm{x}} \mathrm{Zn}_{1-\mathrm{x}} \mathrm{Fe}_{2} \mathrm{O}_{4}$ nanoparticles for hyperthermia. Appl Phys Lett. 2010;96:202511.

23. Beji Z, Hanini A, Smiri LS, et al. Magnetic properties of Zn-substituted $\mathrm{MnFe}_{2} \mathrm{O}_{4}$ nanoparticles synthesized in polyol as potential heating agents for hyperthermia. Evaluation of their toxicity on endothelial cells. Chem Mater. 2010;22:5420-5429.

24. Lee JH, Jang JT, Choi JS, et al. Exchange-coupled magnetic nanoparticles for efficient heat induction. Nat Nanotechnol. 2011;6:418-422.

25. Rodrigues HF, Mello FM, Branquinho LC, Zufelato N, SilveiraLacerda EP, Bakuzis AF. Real-time infrared thermography detection of magnetic nanoparticle hyperthermia in a murine model under a non-uniform field configuration. Int J Hyperthermia. 2013;29(8):752-767. 
26. Bunce NH, Moon JC, Bellenger NG, et al. Improved cine cardiovascular magnetic resonance using Clariscan (NC100150 injection). J Cardiovasc Magn Reson. 2001;3:303-310.

27. Wagner M, Wagner S, Schnorr J, et al. Coronary MR Angiography Using Citrate-Coated Very Small Superparamagnetic Iron Oxide Particles as Blood-Pool Contrast Agent: Initial Experience in Humans. J Magn Reson Imaging. 2011;34:816-823.

28. Iversen NK, Frische S, Thomsen K, et al. Superparamagnetic iron oxide polyacrylic acid coated $\gamma-\mathrm{Fe}_{2} \mathrm{O}_{3}$ nanoparticles do not affect kidney function but cause acute effect on the cardiovascular function in healthy mice. Toxicol Appl Pharmacol. 2013;266:276-288.

29. Sosnovik DE, Nahrendorf M, Weissleder R. Molecular magnetic resonance imaging in cardiovascular medicine. Circulation. 2007;115: 2076-2086.

30. Verde EL, Landi GT, Carrião MS, et al. Field dependent transition to the non-linear regime in magnetic hyperthermia experiments: Comparison between maghemite, cooper, zinc, nickel and cobalt ferrite nanoparticles of similar sizes. AIP Adv. 2012;2:032120.

31. Gratton SE, Ropp PA, Pohlhaus PD, et al. The effect of particle design on cellular internalization pathways. Proc Natl Acad Sci U S A. 2008; 105:11613-11618.

32. Itri R, Depeyrot J, Tourinho FA, Sousa MH. Nanoparticle chain-like formation in eletrical double-layered magnetic fluids evidenced by small-angle X-ray scattering. The European Physical Journal E. 2001;4: 201-208

33. Gomes JA, Sousa MH, Tourinho FA, et al. Synthesis of core-shell ferrite nanoparticles for ferrufluids: Chemical and magnetic analysis. J Phys Chem C. 2008;112(6):6220-6227.

34. Apopa PL, Qian Y, Shao R, et al. Iron oxide nanoparticles induce human microvascular endothelial cell permeability through reactive oxygen species production and microtubule remodeling. Part Fibre Toxicol. 2009;6:1.

35. Brurok H, Berg K, Sneen L, Grant D, Karlsson JO, Jynge P. Cardiac metal contents after infusions of manganese. An experimental evaluation in the isolated rat heart. Invest Radiol. 1999;34:470-476.

36. Safi M, Courtois J, Seigneuret M, Conjeaud H, Berret JF. The effects of aggregation and protein corona on the cellular internalization of iron oxide nanoparticles. Biomaterials. 2011;32:9353-9363.
37. Bregar VB, Lojk J, Sustar V, Suštar V, Veranič P, Pavlin M. Visualization of internalization of functionalized cobalt ferrite nanoparticles and their intracellular fate. Int J Nanomedicine. 2013;8:919-931.

38. Wu X, Tan Y, Mao H, Zhang M. Toxic effects of iron oxide nanoparticles on human umbilical vein endothelial cells. Int J Nanomedicine. 2010;5:385-399.

39. Bers DM. Calcium fluxes involved in control of cardiac myocyte contraction. Circ Res. 2000;87:275-281.

40. Silva AC, Bock NA. Manganese-enhanced MRI: an exceptional tool in translational neuroimaging. Schizophr Bull. 2008;34:595-604.

41. Decuzzi P, Ferrari M. The role of specific and non-specific interactions in receptor-mediated endocytosis of nanoparticles. Biomaterials. 2007;28:2915-2922.

42. Decuzzi P, Ferrari M. The receptor-mediated endocytosis of nonspherical particles. Biophys J. 2008;94:3790-3797.

43. Decuzzi P, Pasqualini R, Arap W, Ferrari M. Intravascular delivery of particulate systems: Does geometry really matter? Pharm Res. 2009; 26(1):235-243.

44. Rosas-Hernández H, Jimenez-Badillo S, Martinez-Cuevas PP, et al. Effects of 45-nm silver nanoparticles on coronary endothelial cells and isolated rat aortic rings. Toxicol Lett. 2009;191:305-313.

45. Frame MD, Dewar AM, Mullick Chowdhury S, Sitharaman B. Vasoactive effects of stable aqueous suspensions of single walled carbon nanotubes in hamsters and mice. Nanotoxicology. 2014;8(8):867-875.

46. Choi HS, Liu W, Misra P, et al. Renal clearance of quantum dots. Nat Biotechnol. 2007;25:1165-1170.

47. Wunderbaldinger P, Josephson L, Weissleder R. Tat peptide directs enhanced clearance and hepatic permeability of magnetic nanoparticles. Bioconjug Chem. 2002;13:264-268.

48. Krombach GA, Wendland MF, Higgins CB, Saeed M. MR imaging of spatial extent of microvascular injury in reperfused ischemically injured rat myocardium: value of blood pool ultrasmall superparamagnetic particles of iron oxide. Radiology. 2002;225:479-486.

49. Monopoli MP, Aberg C, Salvati A, Dawson KA. Biomolecular coronas provide the biological identity of nanosized materials. Nat Nanotechnol. 2012;7(12):779-786. 


\section{Supplementary material}

Table SI Basal values of mean arterial pressure and heart rate of the dose-response curve for magnetic nanoparticles

\begin{tabular}{llr}
\hline Sample & MAP (mmHg) & HR (bpm) \\
\hline CiMNPs & $110.9 \pm 6.80$ & $323.2 \pm 39.8$ \\
PhMNPs & $104.6 \pm 4.04$ & $357.2 \pm 13.0$ \\
BaMNPs & $105.1 \pm 5.04$ & $350.5 \pm 73.8$ \\
\hline
\end{tabular}

Note: Values are shown as the mean \pm standard error.

Abbreviations: BaMNPs, bare magnetic nanoparticles; CiMNPs, citrate-coated magnetic nanoparticles; HR, heart rate; MAP, mean arterial pressure; PhMNPs, tripolyphosphate-coated magnetic nanoparticles.

\section{Publish your work in this journal}

The International Journal of Nanomedicine is an international, peerreviewed journal focusing on the application of nanotechnology in diagnostics, therapeutics, and drug delivery systems throughout the biomedical field. This journal is indexed on PubMed Central, MedLine, CAS, SciSearch ${ }^{\circledR}$, Current Contents ${ }^{\circledR} /$ Clinical Medicine,
Journal Citation Reports/Science Edition, EMBase, Scopus and the Elsevier Bibliographic databases. The manuscript management system is completely online and includes a very quick and fair peer-review system, which is all easy to use. Visit http://www.dovepress.com/ testimonials.php to read real quotes from published authors.

Submit your manuscript here: http://www.dovepress.com/international-journal-of-nanomedicine-journal 